\title{
Development of EST-based SNP and InDel markers and their utilization in tetraploid cotton genetic mapping
}

\author{
Ximei Li ${ }^{1,2}$, Wenhui Gao ${ }^{1}$, Huanle Guo ${ }^{1}$, Xianlong Zhang ${ }^{1}$, David D Fang ${ }^{3}$ and Zhongxu Lin ${ }^{1 *}$
}

\begin{abstract}
Background: Availability of molecular markers has proven to be an efficient tool in facilitating progress in plant breeding, which is particularly important in the case of less researched crops such as cotton. Considering the obvious advantages of single nucleotide polymorphisms (SNPs) and insertion-deletion polymorphisms (InDels), expressed sequence tags (ESTs) were analyzed in silico to identify SNPs and InDels in this study, aiming to develop more molecular markers in cotton.
\end{abstract}

Results: A total of 1,349 EST-based SNP and InDel markers were developed by comparing ESTs between Gossypium hirsutum and G. barbadense, mining G. hirsutum unigenes, and analyzing $3^{\prime}$ untranslated region ( $3^{\prime} \cup T R$ ) sequences. The marker polymorphisms were investigated using the two parents of the mapping population based on the single-strand conformation polymorphism (SSCP) analysis. Of all the markers, 137 (10.16\%) were polymorphic, and revealed 142 loci. Linkage analysis using a $\mathrm{BC}_{1}$ population mapped 133 loci on the 26 chromosomes. Statistical analysis of base variations in SNPs showed that base transitions accounted for $55.78 \%$ of the total base variations and gene ontology indicated that cotton genes varied greatly in harboring SNPs ranging from 1.00 to 24.00 SNPs per gene. Sanger sequencing of three randomly selected SNP markers revealed discrepancy between the in silico predicted sequences and the actual sequencing results.

Conclusions: In silico analysis is a double-edged blade to develop EST-SNP/InDel markers. On the one hand, the designed markers can be well used in tetraploid cotton genetic mapping. And it plays a certain role in revealing transition preference and SNP frequency of cotton genes. On the other hand, the developmental efficiency of markers and polymorphism of designed primers are comparatively low.

Keywords: Cotton, Molecular markers, SNP, InDel, Genetic mapping

\section{Background}

Molecular markers are the foundation of modern molecular plant breeding. There are many types of molecular markers such as restriction fragment length polymorphism (RFLP), simple sequence repeat (SSR) and SNPs. In cotton which is the world most important natural fiber crop, the most prevalent marker type as of today is SSR. However, with the advent of next generation sequencing technologies that significantly reduce sequencing cost,

\footnotetext{
* Correspondence: linzhongxu@mail.hzau.edu.cn

'National Key Laboratory of Crop Genetic Improvement \& National Centre of Plant Gene Research (Wuhan), Huazhong Agricultural University, Wuhan 430070, Hubei, China

Full list of author information is available at the end of the article
}

SNP markers are becoming more and more important due to their abundance in the genome and very simple genetic mode (bi-allelic).

Cotton researchers have tried different methods to develop SNP markers. An et al. [1] reported a few SNP markers when studying R2R3-MYB transcription factors. In 2009, Van Deynze [2] reported the first large-scale SNP discovery results in cotton. They developed about 1,000 SNPs and $300 \mathrm{InDels}$ by re-sequencing the ESTs of 24 upland cotton genotypes. About 200 of these SNPs were also mapped in the TM-1 $\times$ 3-79 genetic map $[3,4]$. Recently, research in cotton SNP discovery has been accelerated and many SNP markers have been reported

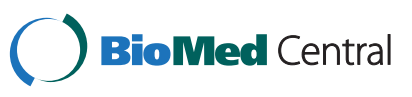

(c) 2014 Li et al.; licensee BioMed Central Ltd. This is an Open Access article distributed under the terms of the Creative Commons Attribution License (http://creativecommons.org/licenses/by/4.0), which permits unrestricted use, distribution, and reproduction in any medium, provided the original work is properly credited. The Creative Commons Public Domain Dedication waiver (http://creativecommons.org/publicdomain/zero/1.0/) applies to the data made available in this article, unless otherwise stated. 
$[5,6]$. In spite of this, the number of cotton SNP markers is still low as compared with other major crops such as maize or soybean. More importantly, a great majority of these SNP markers have neither been validated in other genotypes nor mapped.

Due to its allotetraploid nature, it has been a challenge to differentiate a true SNP (within a sub-genome) from a pseudo-SNP (between subgenomes) in cotton SNP marker development. In 2009, Trick et al. [7] developed many SNP markers in Brassica napus that is also an allotetraploid using transcriptome sequencing. This study provided some insights that could be useful in the development of cotton SNP markers.

Direct sequencing has been a standard method to develop SNP markers, although its efficiency is low, especially in plants with complex genomes $[8,9]$. Another alternative is to take advantage of the large amount of sequence data available in public databases to develop SNP and InDel markers using bioinformatics [10].

Expressed sequence tags (ESTs) have been mined for large-scale SNP discovery in plants including Arabidopsis [11], barley [12], maize [13], sugarcane [14], tomato [15], and cotton [2]. SNPs mined from ESTs have the potential to be functional markers if the particular EST or gene is responsible for phenotypic variations [16,17]. Several methods for identifying SNPs from ESTs have been reported $[17,18]$, and numerous cotton ESTs are available in public databases [19], providing important foundations for the development of EST-based cotton SNP markers.

The 3' untranslated regions (UTRs) undergo less selective pressure than the coding sequences (CDSs) [20], resulting in a higher rate of sequence variation than the CDSs. Thus, 3'UTRs have become valuable resources in identifying SNPs or InDels, especially in those species with duplicate genomes [21]. Koepke et al. [22] developed InDel markers by focusing on the 3'UTRs of the RNA-seq data in sweet cherry.

In the present study, cotton SNP and InDel markers were developed using four strategies. First, interspecific EST-SNPs were developed by comparing the ESTs between G. hirsutum and G. barbadense. Second, intraspecific EST-SNPs were developed by mining the unigenes of G. hirsutum. Third, EST-InDels were developed by mining the 3'UTRs of public G. hirsutum sequences. And fourth, InDel markers were developed by blasting putative 3'UTRs of G. hirsutum against the 3'UTRs of Arabidopsis. Subsequently, we used SSCP technology to validate these markers, and analyzed their polymorphisms between the two mapping parents. Polymorphic markers were used to genotype our $\mathrm{BC}_{1}$ mapping population [19] and mapped. SNP and InDel markers developed in this report will be a valuable genomic resource for cotton genetics and breeding research.

\section{Results}

In silico analysis and primer design Interspecific EST-SNP markers

The collected 273,779 G. hirsutum ESTs and 11,311 G. barbadense ESTs were clustered into 3,263 clusters, which were then imported into HaploSNPer (http://www. bioinformatics.nl/tools/haplosnper/) to identify the interspecific SNPs. Of the 3,263 clusters, 1,668 (51.12\%) had no SNPs, 109 (3.34\%) had only inter-homoeologous SNPs, 200 (6.13\%) had inter/hemi-SNPs, and 1,286 (39.41\%) had hemi-SNPs. Only the clusters containing inter/hemi-SNPs or hemi-SNPs were used to design primers to detect interspecific SNPs (see Additional files 1 and 2).

Among the 200 clusters containing inter/hemi-SNPs, the number of clusters containing $4,5,6,7$ and $\geq 8$ sequences was $46,28,24,15$ and 87 , respectively. Five clusters were removed due to failing to meet the stringent criteria described in the 'Methods'. Among the remaining 195 clusters, the number of clusters containing 4, 5, 6, 7 and $\geq 8$ sequences were 43 (93.48\%), 27 (96.43\%), 23 (95.83\%), 15 (100.00\%) and 87 (100.00\%), respectively. Eventually, 27, 21, 16, 11 and 59 inter/ hemi-SNPs, and 0, 2, 1, 2 and 10 hemi-SNPs were developed, respectively (see Additional file 3). Detailed information of the markers, sequence accession numbers and sequences used to design primers, and SNPs of 134 inter/hemi-SNPs and 15 hemi-SNPs are listed in the Additional file 1.

To those 1,286 clusters containing only hemi-SNPs, the same classification analysis was conducted. After selection of the same stringent criteria, only 276 (21.46\%) clusters were kept for SNP marker design. Finally, a total of 207 hemi-SNPs were developed (see Additional files 1 and 4).

\section{Intraspecific EST-SNP markers}

There were a total of 21,738 unigenes available from NCBI (http://www.ncbi.nlm.nih.gov/) in July 2010 when this research was initiated, derived from more than 19 cotton genotypes. After removing 7,449 unigenes containing less than 4 sequences, the remaining 14,289 unigenes were downloaded from NCBI, and the 1,339 unigenes containing sequences originating from the same genotype were further removed. As a result, 12,950 unigenes were imported into HaploSNPer to identify EST-SNPs. The results showed that 4,378 unigenes did not contain SNPs, while 8,572 unigenes contained SNPs.

The 8,572 unigenes were first classified according to the previously mentioned standards. Then the unigenes containing only putative SNPs were removed, and only 475 (5.54\%) unigenes containing reliable SNPs were kept for primer design. Finally, 455 intraspecific EST-SNP markers 
were designed (see Additional files 5 and 6). In general, one primer pair was developed from each unigene. However, ideal primer pair could not be designed from some unigenes; and two or more primer pairs were designed from the unigenes containing some distant SNPs to amplify more possible SNPs.

\section{InDel markers of $G$. hirsutum}

A total of $8,938 \mathrm{G}$. hirsutum nucleiotide sequences were evaluated for possible InDel marker development, and 1,021 sequences with complete CDSs were selected. Among these sequences, 615 had 3'UTR sequences longer than $100 \mathrm{bp}$. After removing redundancy, 509 unique sequences were eligible to develop InDel markers. In total, 415 HAU-InDel-prefixed markers were developed (see Additional file 7).

Three sets of G. hirsutum sequences, including 65,520 genome survey sequences (GSS), 15,815 nucleotides sequences and 65,371 mRNAs were used to blast against the 25,843 3'UTRs of Arabidopsis sequences. According to the criteria described in the 'Methods', 8, 107 and 218 sequences were homologous to the 3'UTRs of Arabidopsis sequences including 111 singlets and 62 contigs. Subsequently, another $123 \mathrm{HAU}-\mathrm{InDel}$-prefixed markers were developed (see Additional files 8 and 9).

\section{Polymorphisms of the SNP and InDel markers}

All the 1,349 SNP and InDel markers described above were analyzed for their polymorphisms between G. hirsutum cv. Emian22 and G. barbadense acc. 3-79 using SSCP method. As a result, 137 (10.16\%) primer pairs were polymorphic, and produced 142 loci (Table 1).

Among the 356 interspecific EST-SNP primer pairs, 47 (13.20\%) pairs were polymorphic, and revealed 50 loci, including 23 inter/hemi-SNPs and 24 hemi-SNPs (Table 1). The 23 polymorphic inter/hemi-SNPs were screened out of 134 primer pairs, with a polymorphic rate of $17.16 \%$. And the 24 polymorphic hemi-SNPs were selected from 222 primer pairs, with a polymorphic rate of $10.81 \%$. SSCP analysis revealed 43 $(9.45 \%)$ polymorphic markers out of the total 455 intraspecific EST-SNP primer pairs, producing 43 loci (Table 1). As for the polymorphisms of the InDel markers, 47 were polymorphic, and revealed 49 loci. More specifically, of the total 415 InDel markers designed through G. hirsutum mRNAs, 41 (9.88\%) were polymorphic, and produced 42 loci (Table 1). Among the total 123 InDel markers developed from blast analysis against Arabidopsis 3'UTRs, 6 (4.88\%) were polymorphic, and produced 7 loci (Table 1).

Table 1 Polymorphic rates of the SNP and InDel markers

\begin{tabular}{|c|c|c|c|c|c|c|c|c|}
\hline Primers & No. markers & $\begin{array}{l}\text { No. polymorphic } \\
\text { markers/loci }\end{array}$ & $\begin{array}{l}\text { Polymorphic } \\
\text { rate }(\%)\end{array}$ & Classes & Subclasses $^{5)}$ & No. Markers & $\begin{array}{l}\text { No. Polymorphic } \\
\text { markers/loci }\end{array}$ & $\begin{array}{l}\text { Polymorphic } \\
\text { rate (\%) }\end{array}$ \\
\hline \multirow[t]{10}{*}{ HAU-SNP1) } & 356 & $47 / 50$ & 13.20 & & 4 & 27 & $6 / 6$ & 22.22 \\
\hline & & & & inter & 5 & 21 & $4 / 5$ & 19.05 \\
\hline & & & & $23 / 134$ & 6 & 16 & $2 / 3$ & 12.50 \\
\hline & & & & $17.16 \%$ & 7 & 11 & $0 / 0$ & 0.00 \\
\hline & & & & & $\geq 8$ & 59 & $11 / 11$ & 18.64 \\
\hline & & & & & 4 & 0 & $0 / 0$ & - \\
\hline & & & & hemi & 5 & 8 & $1 / 1$ & 12.50 \\
\hline & & & & $24 / 222$ & 6 & 9 & $2 / 2$ & 22.22 \\
\hline & & & & $10.81 \%$ & 7 & 10 & $0 / 0$ & 0.00 \\
\hline & & & & & $\geq 8$ & 195 & $21 / 22$ & 10.77 \\
\hline \multirow[t]{5}{*}{ HAU-SNP2) } & 455 & $43 / 43$ & 9.45 & & 4 & 171 & $15 / 15$ & 8.77 \\
\hline & & & & & 5 & 89 & $12 / 12$ & 13.48 \\
\hline & & & & & 6 & 97 & $6 / 6$ & 6.19 \\
\hline & & & & & 7 & 38 & $3 / 3$ & 7.89 \\
\hline & & & & & $\geq 8$ & 60 & $7 / 7$ & 11.67 \\
\hline HAU-InDe $\left.\right|^{3)}$ & 415 & $41 / 42$ & 9.88 & & & & & \\
\hline HAU-InDel| ${ }^{4)}$ & 123 & $6 / 7$ & 4.88 & & & & & \\
\hline Total & 1,349 & $137 / 142$ & 10.16 & & & & & \\
\hline
\end{tabular}

1) HAU-SNP001 356, which were developed by comparing ESTs between G. hirsutum and G. barbadense.

${ }^{2)} \mathrm{HAU}-\mathrm{SNP} 357 \sim 811$, which were developed by mining $G$. hirsutum unigenes.

${ }^{3)} \mathrm{HAU}-\mathrm{InDel001} \sim 415$, which were developed by mining the 3 'UTRs of public G. hirsutum sequences.

${ }^{4)} \mathrm{HAU}-\mathrm{InDel} 416 \sim 538$, which were developed by blasting putative 3'UTRs of G. hirsutum against the 3'UTRs of Arabidopsis.

${ }^{5)}$ Subclasses mean different types of clusters/unigenes classified by the number of sequences contained in a cluster/unigene. 
Distribution of SNP and InDel markers on the interspecific $\mathrm{BC}_{1}$ linkage map

After linkage analysis, 133 of the 142 SNP and InDel polymorphic loci were mapped on the 26 cotton chromosomes. Sixty-six loci were mapped on the 13 chromosomes of the $\mathrm{A}_{\mathrm{T}}$ genome, which included 1,204 loci with a total genetic distance of 2,297.27 cM and an average marker interval of $1.91 \mathrm{cM}$. Sixty-seven loci were mapped on the 13 chromosomes of the $\mathrm{D}_{\mathrm{T}}$ genome, which included 1,415 loci with a total genetic distance of 2,246.24 cM and an average marker interval of $1.59 \mathrm{cM}$. The present interspecific linkage map contains 2,619 loci with a total genetic distance of $4,543.51 \mathrm{cM}$ and an average marker interval of $1.73 \mathrm{cM}$ (Table 2, and also see
Additional file 10). Although the 133 SNP and InDel markers were mapped on all 26 chromosomes, they were not evenly distributed. Chr09, Chr10, Chr19 and Chr26 had more loci, while Chr04 and Chr06 had fewer loci (Table 2, see Additional file 10).

\section{Statistical analysis of base variations and SNP frequency of cotton genes}

Statistical analysis of reliable base variations showed that the SNPs in different clusters/unigenes that were used to design HAU-SNP-prefixed markers had the same tendency towards more base transitions $(\mathrm{C} \rightarrow \mathrm{T}$ or $\mathrm{G} \rightarrow \mathrm{A})$ (Table 3). Specifically, the percentage of a certain base variation varied from $1.27 \%(\mathrm{C} /-)$ to $28.56 \%(\mathrm{C} \rightarrow \mathrm{T})$ in

Table 2 Distribution of SNP and InDel markers on the interspecific BC $_{\mathbf{1}}$ linkage map

\begin{tabular}{|c|c|c|c|c|c|c|c|c|}
\hline Chromosome & Size (cM) & Marker interval (cM) & Total loci & SNP loci & HAU-SNP loci ${ }^{1)}$ & HAU-SNP loci' ${ }^{2)}$ & HAU-InDel loci ${ }^{3)}$ & HAU-InDel loci ${ }^{4)}$ \\
\hline Chr01 & 186.87 & 2.46 & 76 & 3 & 1 & 2 & 0 & 0 \\
\hline Chr02 & 156.03 & 2.40 & 65 & 6 & 3 & 2 & 1 & 0 \\
\hline Chr03 & 156.23 & 2.06 & 76 & 5 & 2 & 2 & 1 & 0 \\
\hline Chro4 & 140.07 & 2.50 & 56 & 1 & 0 & 0 & 1 & 0 \\
\hline Chr05 & 242.76 & 1.75 & 139 & 6 & 1 & 2 & 3 & 0 \\
\hline Chr06 & 171.43 & 2.04 & 84 & 1 & 1 & 0 & 0 & 0 \\
\hline Chr07 & 103.39 & 1.48 & 70 & 4 & 2 & 2 & 0 & 0 \\
\hline Chr08 & 148.05 & 1.53 & 97 & 4 & 0 & 3 & 1 & 0 \\
\hline Chr09 & 148.83 & 1.43 & 104 & 9 & 2 & 3 & 3 & 1 \\
\hline Chr10 & 179.66 & 1.91 & 94 & 9 & 4 & 2 & 2 & 1 \\
\hline Chr11 & 234.77 & 1.63 & 144 & 7 & 1 & 2 & 4 & 0 \\
\hline Chr12 & 221.04 & 2.19 & 101 & 6 & 3 & 0 & 2 & 1 \\
\hline Chr13 & 208.14 & 2.12 & 98 & 5 & 2 & 2 & 1 & 0 \\
\hline$A_{T}$ genome & 2297.27 & 1.91 & 1204 & 66 & 22 & 22 & 19 & 3 \\
\hline Chr14 & 156.15 & 1.63 & 96 & 4 & 1 & 0 & 2 & 1 \\
\hline Chr15 & 189.00 & 1.60 & 118 & 5 & 2 & 0 & 3 & 0 \\
\hline Chr16 & 94.32 & 0.99 & 95 & 5 & 4 & 1 & 0 & 0 \\
\hline Chr17 & 149.43 & 2.13 & 70 & 5 & 3 & 2 & 0 & 0 \\
\hline Chr18 & 146.95 & 1.47 & 100 & 6 & 3 & 2 & 0 & 1 \\
\hline Chr19 & 252.27 & 1.64 & 154 & 8 & 1 & 4 & 3 & 0 \\
\hline Chr20 & 107.50 & 1.00 & 108 & 6 & 3 & 0 & 2 & 1 \\
\hline Chr21 & 256.03 & 1.82 & 141 & 5 & 1 & 3 & 1 & 0 \\
\hline Chr22 & 166.03 & 1.84 & 90 & 3 & 1 & 0 & 2 & 0 \\
\hline Chr23 & 193.19 & 1.82 & 106 & 4 & 1 & 2 & 1 & 0 \\
\hline Chr24 & 187.04 & 1.64 & 114 & 3 & 1 & 1 & 1 & 0 \\
\hline Chr25 & 151.25 & 1.41 & 107 & 5 & 3 & 1 & 1 & 0 \\
\hline Chr26 & 197.09 & 1.70 & 116 & 8 & 1 & 3 & 3 & 1 \\
\hline $\mathrm{D}_{\text {T }}$ genome & 2246.24 & 1.59 & 1415 & 67 & 25 & 19 & 19 & 4 \\
\hline Total & 4543.51 & 1.73 & 2619 & 133 & 47 & 41 & 38 & 7 \\
\hline
\end{tabular}

1) HAU-SNP001 356

${ }^{2)} \mathrm{HAU}-\mathrm{SNP} 357 \sim 811$.

3) $\mathrm{HAU}-\mathrm{InDel001} \sim 415$.

4) $\mathrm{HAU}-\mathrm{InDel} 416 \sim 538$. 
Table 3 Summary of cotton base variations

\begin{tabular}{|c|c|c|c|c|c|c|c|c|c|c|}
\hline \multirow[t]{2}{*}{ Types of SNPs } & \multicolumn{3}{|c|}{ Interspecific EST-SNPs } & \multicolumn{6}{|c|}{ Intraspecific EST-SNPs } & \multirow[t]{2}{*}{ Total } \\
\hline & Inter/hemi-SNPs & Hemi-SNPs & Subtotal & 4 & 5 & 6 & 7 & $\geq 8$ & Subtotal & \\
\hline $\mathrm{C} \rightarrow \mathrm{T}$ & $265(26.26 \%)$ & $1036(29.21 \%)$ & $1301(28.56 \%)$ & $158(30.10 \%)$ & $153(36.00 \%)$ & $249(27.04 \%)$ & $103(29.94 \%)$ & $170(24.50 \%)$ & $833(28.64 \%)$ & 2134 (28.59\%) \\
\hline $\mathrm{G} \rightarrow \mathrm{A}$ & $271(26.86 \%)$ & $981(27.66 \%)$ & $1252(27.48 \%)$ & 147 (28.00\%) & $120(28.24 \%)$ & $244(26.49 \%)$ & $97(28.20 \%)$ & $170(24.50 \%)$ & 778 (26.74\%) & 2030 (27.19\%) \\
\hline All transitions & $536(53.12 \%)$ & 2017 (56.86\%) & $2553(56.04 \%)$ & 305 (58.10\%) & $273(64.24 \%)$ & 493 (53.53\%) & $200(58.14 \%)$ & 340 (48.99\%) & $1611(55.38 \%)$ & $4164(55.78 \%)$ \\
\hline$C \rightarrow G$ & $74(7.33 \%)$ & $282(7.95 \%)$ & $356(7.81 \%)$ & $40(7.62 \%)$ & $12(2.82 \%)$ & $61(6.62 \%)$ & $17(4.94 \%)$ & 47 (6.77\%) & $177(6.09 \%)$ & $533(7.14 \%)$ \\
\hline $\mathrm{A} \rightarrow \mathrm{T}$ & $82(8.13 \%)$ & $410(11.56 \%)$ & $492(10.80 \%)$ & 45 (8.57\%) & 41 (9.65\%) & $96(10.42 \%)$ & $33(9.59 \%)$ & $61(8.79 \%)$ & $276(9.49 \%)$ & 768 (10.29\%) \\
\hline$C \rightarrow A$ & $88(8.72 \%)$ & $304(8.57 \%)$ & $392(8.60 \%)$ & 33 (6.29\%) & $31(7.29 \%)$ & $67(7.27 \%)$ & $23(6.69 \%)$ & $53(7.64 \%)$ & $207(7.12 \%)$ & $599(8.02 \%)$ \\
\hline $\mathrm{T} \rightarrow \mathrm{G}$ & 97 (9.61\%) & $304(8.57 \%)$ & 401 (8.80\%) & $42(8.00 \%)$ & $32(7.53 \%)$ & 79 (8.58\%) & $19(5.52 \%)$ & $73(10.52 \%)$ & $245(8.42 \%)$ & $646(8.65 \%)$ \\
\hline All transversions & 341 (33.80\%) & $1300(36.65 \%)$ & $1641(36.02)$ & 160 (30.48\%) & 116 (27.29\%) & 303 (32.90\%) & 92 (26.74\%) & 234 (33.72\%) & 905 (31.11\%) & $2546(34.11 \%)$ \\
\hline$A /-$ & $52(5.15 \%)$ & $56(1.58 \%)$ & $108(2.37 \%)$ & $18(3.43 \%)$ & $13(3.06 \%)$ & $40(4.34 \%)$ & $25(7.27 \%)$ & 47 (6.77\%) & $143(4.92 \%)$ & 251 (3.36\%) \\
\hline C/- & $22(2.18 \%)$ & $36(1.01 \%)$ & $58(1.27 \%)$ & $13(2.48 \%)$ & $5(1.18 \%)$ & $29(3.15 \%)$ & $4(1.16 \%)$ & $16(2.31 \%)$ & 67 (2.30\%) & $125(1.67 \%)$ \\
\hline G/- & $28(2.78 \%)$ & $37(1.04 \%)$ & $65(1.43 \%)$ & $16(3.05 \%)$ & $3(0.71 \%)$ & $27(2.93 \%)$ & $11(3.20 \%)$ & $22(3.17 \%)$ & 79 (2.72\%) & $144(1.93 \%)$ \\
\hline T/- & 30 (2.97\%) & $101(2.85 \%)$ & $131(2.88 \%)$ & $13(2.48 \%)$ & 15 (3.53\%) & 29 (3.15\%) & 12 (3.49\%) & 35 (5.04\%) & $104(3.58 \%)$ & 235 (3.15\%) \\
\hline All InDels & $132(13.08 \%)$ & $230(6.48 \%)$ & $362(7.95 \%)$ & $60(11.43 \%)$ & $36(8.47 \%)$ & 125 (13.57\%) & $52(15.12 \%)$ & 120 (17.29\%) & 393 (13.51\%) & 755 (10.11\%) \\
\hline Total & 1009 (100.00\%) & 3547 (100.00\%) & 4556 (100.00\%) & 525 (100.00\%) & 425 (100.00\%) & 921 (100.00\%) & 344 (100.00\%) & 694 (100.00\%) & 2909 (100.00\%) & 7465 (100.00\%) \\
\hline
\end{tabular}


the clusters used to design the interspecific EST-SNP markers, and from $2.30 \%(\mathrm{C} /-)$ to $28.64 \%(\mathrm{C} \rightarrow \mathrm{T})$ in the unigenes used to design the intraspecific EST-SNP markers (Table 3). In total, the percentage varied from $1.67 \%(\mathrm{C} /-)$ to $28.59 \%(\mathrm{C} \rightarrow \mathrm{T})$ in this study, with base transitions $(\mathrm{C} \rightarrow \mathrm{T}$ or $\mathrm{G} \rightarrow \mathrm{A})$ accounting for $55.78 \%$ of the total SNPs (Table 3).

In order to gain more understanding about the relationship between SNPs and gene functions, we conducted functional annotation analyses of the consensus sequences. The number of SNPs/gene involved in cellular component, molecular function and biological process was 13.90, 9.36 and 10.56 respectively for those clusters used to design interspecific EST-SNP markers. In the unigenes used to design the intraspecific EST-SNP markers, a similar analysis showed that the number of SNPs/gene in cellular component, molecular function and biological process was $6.14,6.46$ and 5.21 , respectively. In total, the highest number of SNPs/gene was in the cellular component category (11.96), followed by the molecular function (8.02) and biological process (7.92) categories (Table 4).

$\mathrm{GO}$ analysis of the total 947 consensus sequences on level 3 showed that 28 sequences were assigned to 4 functions in the 'cellular component' category, 199 were assigned to 24 functions in the 'molecular function' category, and 158 were assigned to 32 functions in the 'biological process' category (see Additional file 11). Among these functions, genes belonging to 'killing of cells of other organism' had the maximum SNPs/gene (24.00); while, genes belonging to 'selenium binding', 'circadian rhythm', etc. harbored the minimum SNPs/gene (1.00) (see Additional file 11).

\section{Confirmation of the predicted SNPs}

To validate the SNPs predicted by in silico analysis, the PCR products generated from three polymorphic primer pairs were randomly chosen to be cloned and Sangersequenced. The results showed that the product sizes of

Table $4 \mathrm{GO}$ analysis of consensus sequences used to design the HAU-SNP-prefixed markers on level 1

\begin{tabular}{llccc}
\hline & Functional categories & $\begin{array}{c}\text { Number } \\
\text { of genes }\end{array}$ & $\begin{array}{c}\text { Number } \\
\text { of SNPs }\end{array}$ & SNPs/gene \\
\hline HAU-SNP $^{1)}$ & Cellular component & 21 & 292 & 13.9 \\
& Molecular function & 107 & 1001 & 9.36 \\
& Biological process & 80 & 845 & 10.56 \\
\multirow{2}{*}{ HAU-SNP ${ }^{2)}$} & Cellular component & 7 & 43 & 6.14 \\
& Molecular function & 92 & 594 & 6.46 \\
& Biological process & 78 & 406 & 5.21 \\
Total & Cellular component & 28 & 335 & 11.96 \\
& Molecular function & 199 & 1595 & 8.02 \\
& Biological process & 158 & 1251 & 7.92 \\
\hline
\end{tabular}

${ }^{1)}$ HAU-SNP001 356. ${ }^{2)}$ HAU-SNP357 811. two markers (HAU-SNP304 and HAU-SNP504) were not different between Emian22, 3-79 and the original sequences, but those of marker HAU-SNP248 were slightly different between them (Figure 1).

In silico analysis predicted several SNPs in the amplified products of the marker HAU-SNP248, including base transitions at the $88^{\text {th }}$ and $93^{\text {rd }}$ bases, and a series of base variations starting at the $161^{\text {st }}$ base. However, sequence analysis of the two mapping parents differed from the predicted results. Two base transitions appeared at the $61^{\text {st }}$ base (A in Emian $22 \rightarrow \mathrm{G}$ in 3-79) and the $80^{\text {th }}$ base ( $\mathrm{C}$ in Emian22 $\rightarrow \mathrm{T}$ in $3-79$ ). The interspecific differences in the end of the amplified sequences appeared at the $163^{\text {rd }}$ and $164^{\text {th }}$ bases $(-$ in Emian22 $\rightarrow \mathrm{T}$ in 3-79) (Figure 1a).

The three predicted interspecific SNPs in marker HAU-SNP304 included base transitions at the $112^{\text {nd }}$ and $118^{\text {th }}$ bases, and a base transversion at the $148^{\text {th }}$ base. These SNPs were confirmed by the two mapping parents without any discrepancy (Figure $1 \mathrm{~b}$ ).

Only one SNP was predicted in the amplified products of the marker HAU-SNP504, which was a transition at the $62^{\text {nd }}$ base $(\mathrm{C}$ in DW $\rightarrow \mathrm{T}$ in DT). However, both Emian22 and 3-79 contained $T$ at the $62^{\text {nd }}$ base, while the difference between the two parents appeared at the $183^{\text {rd }}$ base (A in Emian22 $\rightarrow \mathrm{G}$ in 3-79). Besides the base transitions at the $62^{\text {nd }}$ and $183^{\text {rd }}$ bases, there was no difference among the four sequences (Figure 1c).

\section{Discussion}

\section{Reliability of SNPs contained in clusters/unigenes}

The in silico analysis of clusters used to design the interspecific EST-SNPs showed that, among the 200 clusters containing inter/hemi-SNPs, 195 (97.50\%) contained reliable SNPs. This value increased with the number of sequences contained in the clusters increased (see Additional file 3). Similar trend was observed in the 1,286 clusters containing hemi-SNPs though the number of reliable SNPs was smaller (see Additional file 4). The reason behind this might be that most of the clusters containing hemi-SNPs had fewer G. barbadense sequences while more G. barbadense sequences existed for those containing inter/hemi-SNPs.

In silico analysis of unigenes used to design the intraspecific EST-SNPs showed that, only 475 (5.54\%) of the total 8,572 unigenes containing putative SNPs harbored reliable SNPs. This number further decreased with the increasing number of sequences contained in the unigenes, ranging from $62.03 \%$ to $0.95 \%$ (see Additional file 6). This might be partially due to different sequences of the same genotype submitted by different researchers, along with the increase of the total number of sequences contained in the unigenes that demands more rigorous comparison. 


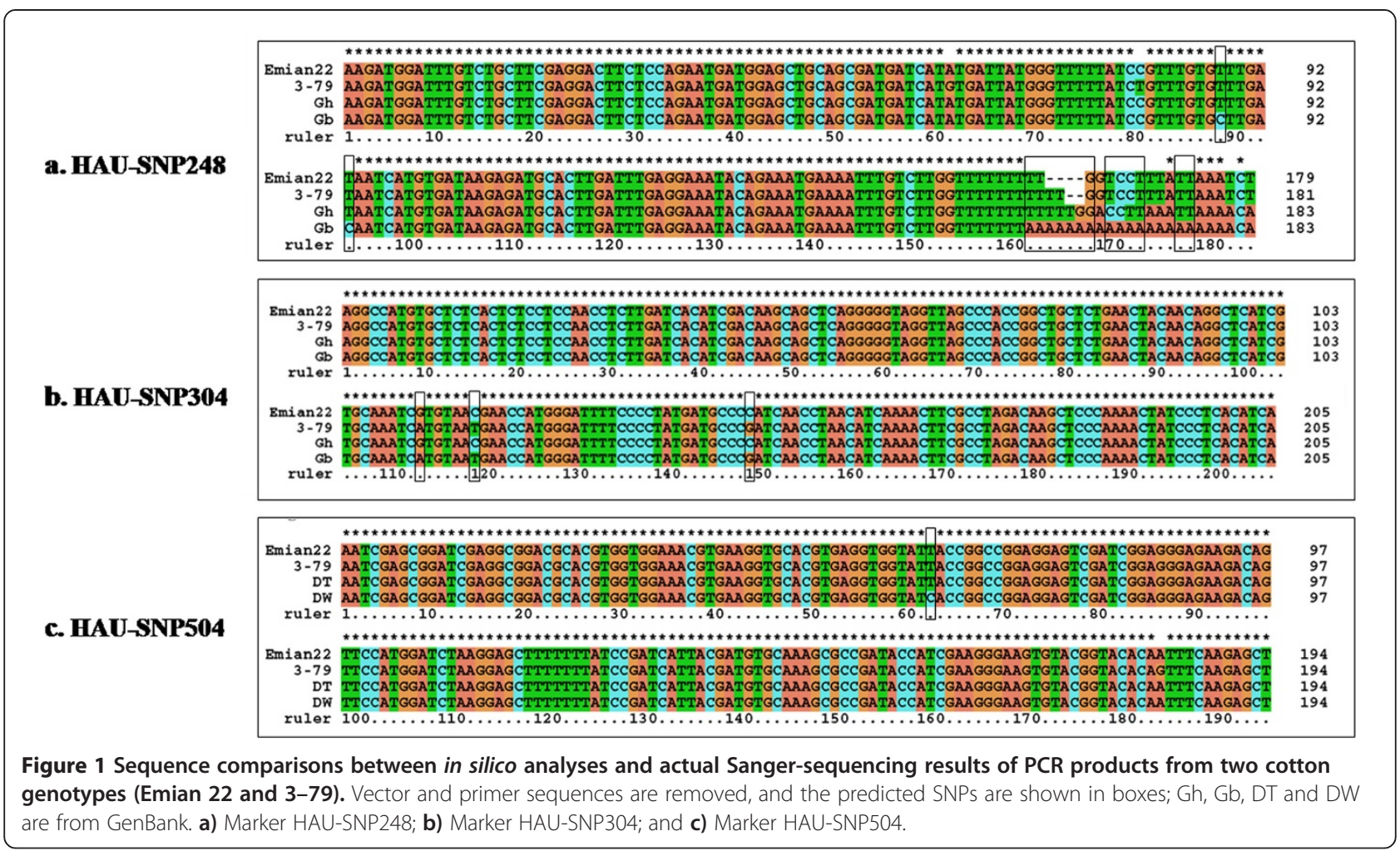

\section{Polymorphism comparison of SNP and InDel markers}

The polymorphic rate of the intraspecific EST-SNP markers $(9.45 \%)$ was relatively low, which is consistent with the fact that markers derived from coding sequences have a lower polymorphism due to their more conserved nature compared to non-coding sequences [23]. The interspecific EST-SNP markers had the highest polymorphic rate $(13.20 \%)$, which is mainly due to the focused attention on the interspecific differences while developing markers [7]. Although various polymorphic rates of markers among the subclasses existed in the both categories, the polymorphic rate of inter/hemi-SNPs $(17.16 \%)$ was much higher than that of hemi-SNPs (10.81\%).

Compared to the intraspecific EST-SNP markers, the first batch of HAU-InDel-prefixed markers had a higher polymorphic rate $(9.88 \%)$, which is consistent with the results obtained by $\mathrm{Zhu}$ et al. [8]. However, the second batch of HAU-InDel-prefixed markers showed the lowest polymorphic rate (4.88\%). Generally, this is a possible but unsatisfactory method to develop cotton markers by comparing with Arabidopsis sequences. Application of this method might result in inaccurate information. This inaccuracy may be overcome when tetraploid cotton genome sequence becomes available. In summary, appropriate methods of marker development, either through improving the reliability of predicted SNPs or targeting regions possessing more variations, are necessary to increase polymorphic rates.

\section{Even distribution of SNP and InDel markers on the interspecific $\mathrm{BC}_{1}$ linkage map}

No obvious difference was observed in the number of SNP/InDel loci mapped between the $A_{T}$ and $D_{T}$ subgenomes. However, uneven distribution was present among chromosomes. Within a chromosome, the SNP/ InDel loci were relatively evenly distributed (Table 2 , see Additional file 10). The results are consistent with the fact that base substitutions exist throughout the cotton genome [24].

\section{Transition preference and SNP frequency of cotton genes} Statistical analysis of the cotton base variations showed that base transitions appeared more frequently than other base variations, consistent with previous reports of the preference for base transitions $[13,25,26]$. Specifically, base transitions accounted for $55.78 \%$ of all the reliable SNPs in this study, which may be due to methylated cytosines in $\mathrm{CpG}$ dinucleotides changed into thymines during the genesis of the SNPs [27].

SNP frequency of cotton genes on level 1 showed that SNPs/gene decreased gradually in the order of cellular component category, molecular function category and 
biological process category (Table 4). On level 3, SNP frequency in each gene varied from 1.00 to 24.00 among genes with different functions (see Additional file 11). These results could provide directions to the research on SNP effects on gene functions.

\section{Advantages and disadvantages of developing EST-SNP/InDel markers}

With the availability of large number of ESTs and the release of plant genomes, a large number of SNPs/InDels in various plants have been discovered using bioinformatics $[11,13,15]$, indicating that bioinformatics is an efficient tool to discover SNPs/InDels. In this study, however, there were some differences between the predicted results and the sequencing results (Figure 1). The materials used for sequencing in our study are different from those materials for predicting SNPs/InDels, which may account for the differences observed. Additionally, the deviation between bioinformatics and experiments partly explained why these markers had such low polymorphism in this study. In conclusion, direct sequencing of the mapping parents may be the best way to develop highly reliable and polymorphic SNP/InDel markers.

\section{Conclusions}

A total of 1,349 SNP/InDel markers were developed from a large number of ESTs. Of them, 137 markers (10.16\%) were polymorphic between two mapping parents and revealed 142 polymorphic loci based on the SSCP analysis. Although the marker discovery efficiency and marker polymorphism were relatively low, linkage analysis mapped 133 loci on the 26 chromosomes, indicating that EST-based SNPs and InDels developed by in silico analysis are useful in tetraploid cotton genetic mapping. In addition, this study also revealed the preference of base transitions over other types of base variations and different SNP frequencies contained in cotton genes. Sanger sequencing showed certain discrepancy between the in silico sequence prediction and the actual sequences. In general, the in silico analysis is a complementary but of low efficiency method to develop SNPs and InDels in cotton, indicating that resequencing or high-throughput sequencing may be a better way to develop cotton SNPs/ InDels.

\section{Methods}

\section{Plant materials}

Gossypium hirsutum cv. Emian22 and G. barbadense acc. 3-79 were used to detect polymorphisms of the newly developed SNP and InDel markers. The $\mathrm{BC}_{1}$ population [(Emian22 $\times 3-79) \times$ Emian22] with 141 progenies [19] was used as the mapping population to map all polymorphic markers.

\section{In silico analysis and primer design Interspecific EST-SNP markers}

The ESTs of G. hirsutum and G. barbadense downloaded from NCBI (http://www.ncbi.nlm.nih.gov/) were clustered using the wcd program [28]. All of the clusters were then imported into HaploSNPer (http://www.bioinformatics.nl/ tools/haplosnper/) to identify SNPs between G. hirsutum and $G$. barbadense with default parameters. In this step, the clusters containing no SNPs or inter-homoeologous SNPs (Figure 2a) were discarded, while the clusters containing inter/hemi-SNPs (Figure 2b) or hemi-SNPs (Figure 2c) were kept. To be qualified for primer design, the clusters must contain two or more sequences from G. hirsutum and G. barbadense, respectively. Primer 3 (version 0.4.0) (http://frodo.wi.mit.edu/primer3/) was used to design primers with criteria as follows: primer length 18-24 bp, optimum 20 bp; GC content 40-60\%, optimum $50 \%$; optimum annealing temperature $58^{\circ} \mathrm{C}$; and PCR product size 100-300 bp. The SNP markers developed were named as HAU-SNP001 HAU-SNP356.

\section{Intraspecific EST-SNP markers}

G. hirsutum unigenes with at least four ESTs were downloaded from NCBI, and then unigenes with ESTs from the same genotype were discarded. For the remaining unigenes, HaploSNPer was used to identify SNPs with default parameters. Unigenes that contained only potential SNPs predicted by HaploSNPer were further removed (Figure 3a), while unigenes containing reliable SNPs (Figure $3 \mathrm{~b}$ ) were used to design primers with criteria same as those of the interspecific EST-SNP primers. The SNP markers developed herein were named as HAU-SNP357 HAU-SNP811.

\section{InDel markers of G. hirsutum}

Message RNA sequences with complete CDSs were selected from the collected G. hirsutum nucleiotide sequences. After removing redundancy, the remaining unique sequences that had 3'UTR sequences longer than 100 bp were used to develop HAU-InDel-prefixed markers to amplify InDels existing in the 3'UTRs. The criteria for primer design using Primer Premier 6.0 software (http://www. premierbiosoft.com) were as follows: primer length 18-24 bp, optimum 20 bp; GC content 35-60\%, optimum $50 \%$; optimum annealing temperature $55^{\circ} \mathrm{C}$; and PCR product size 100-500 bp [29].

The 3'UTR sequences of Arabidopsis were kindly provided by Prof. Graziano Pesole (graziano.pesole@biologia. uniba.it). The collected genome survey sequences, nucleotide sequences and mRNA sequences of G. hirsutum were used to blast against the Arabidopsis 3'UTR sequences with $E$ value of $1.0 \times \mathrm{E}^{-10}$ and matched sequence length of $\geq 100 \mathrm{bp}$. Subsequently, the redundant sequences were removed manually. The matched parts of the G. hirsutum 


\begin{tabular}{|c|c|c|c|c|c|c|c|c|c|c|}
\hline \multicolumn{2}{|c|}{ a. Haplotype ID s } & sequence name & 171 & 234 & 237 & 256 & 288 & 317 & 326 & 371 \\
\hline 1 & $\underline{G b 92}$ & $299 \quad 315072504$ & $\mathrm{~T}$ & $\mathrm{C}$ & $\mathrm{T}$ & c & A & A & $\mathbf{A}$ & $\mathbf{T}$ \\
\hline 1 & Gh20 & 0456084150638 & . & . & . & . & . & A & $\mathbf{A}$ & $\mathbf{T}$ \\
\hline 1 & Gh24 & 4681973943648 & $T$ & C & $\mathrm{T}$ & c & A & A & $\mathbf{A}$ & $\mathbf{T}$ \\
\hline 2 & $\underline{G b 21}$ & $106 \quad 66783460$ & c & $\mathbf{T}$ & A & A & c & G & G & c \\
\hline 2 & Gh10 & $01588 \quad 164257407$ & c & $\mathbf{T}$ & A & A & c & G & G & c \\
\hline b. Haplotype & eID $s$ & sequence name & locat & ion & 222 & 235 & 2463 & 30633 & 383 & 370 \\
\hline 1 & & Gb2262 66783616 & & & C & A & $\mathrm{T}$ & G & & - \\
\hline 1 & & $\underline{G b 524566786599}$ & & & C & A & $\mathbf{T}$ & G & & - \\
\hline 1 & & Gh61385 164317270 & & & C & A & $\mathbf{T}$ & $\mathrm{T}$ & & - \\
\hline 2 & & Gh135354 109872022 & & & $\mathrm{~T}$ & C & C & C T & & A \\
\hline 2 & & Gh168759 109838610 & & & $\mathbf{T}$ & C & C & $\mathbf{T}$ & & A \\
\hline \multicolumn{2}{|c|}{ c. Haplotype ID } & sequence name & SNP & loca & ation & 596 & 629 & 9635 & & 46 \\
\hline 1 & & Gb2513 66783867 & & & & $\mathbf{T}$ & G & $\mathbf{T}$ & & G \\
\hline 1 & & Gb504 66781858 & & & & $\mathbf{T}$ & G & $\mathbf{T}$ & & G \\
\hline 1 & & Gh251019 73862334 & & & & $\mathbf{T}$ & G & $\mathbf{T}$ & & G \\
\hline 2 & & Gh125253 109882129 & & & & C & C & C & & $\mathbf{T}$ \\
\hline 2 & & Gh279199 31406595 & & & & c & C & C & & $\mathbf{T}$ \\
\hline
\end{tabular}

Figure 2 Three types of clusters during in silico analysis of interspecific EST-SNPs. a: Clusters with only inter-homoeologue SNPS (Both G. hirsutum and G. barbadense harbor two base types at a certain base, and no difference in base types exists between them); b: Clusters with inter/ hemi-SNPs (G. hirsutum and G. barbadense harbor different base types at one or more bases); c: Clusters with only hemi-SNPs (One of G. hirsutum and $\mathrm{G}$. barbadense harbors only one base type at a certain base, and the other one harbors two base types at the certain base. Base types between them are partially different).

\begin{tabular}{|ccccc|}
\hline a. Haplotype ID & sequence name & SNP location & 449 & 507 \\
1 & $\underline{\text { DT567004 }}$ & T & T \\
1 & $\underline{\text { DW230729 }}$ & T & T \\
1 & $\underline{\text { ES831757 }}$ & T & T \\
& 2 & $\underline{\text { DW497812 }}$ & A & C \\
& 2 & $\underline{\text { DW497813 }}$ & A & C \\
\hline b. & Haplotype ID & sequence name & SNP location & 110 \\
& 1 & $\underline{\text { DN780229 }}$ & \multicolumn{2}{c|}{ G } \\
& 1 & $\underline{\text { DN780234 }}$ & \multicolumn{2}{c|}{ G } \\
& 2 & $\underline{\text { DW488976 }}$ & \multicolumn{2}{c|}{ T } \\
& 2 & $\underline{\text { DW488977 }}$ & \multicolumn{2}{c|}{ T } \\
\hline
\end{tabular}

Figure 3 Two types of unigenes during in silico analysis of intraspecific EST-SNPs. a: Unigenes with only putative SNPs; $\mathbf{b}$ : Unigenes with reliable SNPS. 
sequences were also used to design HAU-InDel-prefixed primers following the similar methods described above.

\section{Genotyping markers using SSCP analysis}

PCR amplification of SNP and InDel markers was conducted according to the methods described by Lin et al. [30]. Polymorphism detection between the two mapping parents and genotyping of the whole mapping population using polymorphic markers were carried out according to the improved SSCP technology described by $\mathrm{Li}$ et al. [31]. In brief, the amplified products were denatured in a boiling water bath for five minutes. The single-stranded DNA was separated on an $8 \%$ native polyacrylamide gel (29 acrylamide: $1 \mathrm{~N}, \mathrm{~N}$-methylene bisacrylamide) at a constant watt of $15 \mathrm{~W}$ for about $4 \mathrm{~h}$, and DNA fragments were detected with silver staining (an example see Additional file 12).

\section{Genetic mapping}

The polymorphic SNP and InDel markers were integrated into our previously published interspecific $\mathrm{BC}_{1}$ linkage map [19,29,31,32]. The logarithm of odds (LOD) threshold during map construction was 8.0, while the other parameters were the same as those described by Li et al. [31].

\section{Statistical analysis of base variations and SNP frequency of cotton genes}

All the reliable SNPs were subjected to the statistical analysis, producing six kinds of base variations including $\mathrm{A} /$ - or $\mathrm{T} /$-, $\mathrm{C} /$ - or $\mathrm{G} /-, \mathrm{A} \rightarrow \mathrm{G}$ or $\mathrm{T} \rightarrow \mathrm{C}, \mathrm{A} \rightarrow \mathrm{C}$ or $\mathrm{T} \rightarrow$ $\mathrm{G}, \mathrm{A} \rightarrow \mathrm{T}$ or $\mathrm{T} \rightarrow \mathrm{A}$ and $\mathrm{C} \rightarrow \mathrm{G}$ or $\mathrm{G} \rightarrow \mathrm{C}$. In addition, the SNP frequency in cotton genes was evaluated by combining the gene ontology analyses of all the sequences used to design the HAU-SNP-prefixed markers. The functional annotation of nucleotide sequences was performed using Blast2GO [33,34] with default parameters, and the subsequent analyses were conducted according to the methods described by Li et al. and annotations on level 3 were directly used [35].

\section{Validation of predicted SNPs using Sanger sequencing}

The PCR-amplified products of randomly chosen SNP markers were recovered from agarose gels and purified using QIAGEN purification kits (QIAGEN, Dusseldorf, Germany). The purified amplicons were cloned into T-Easy vector (Promega, Madison, Wis., USA). Then at least three clones were randomly selected to be commercially sequenced from both ends using M13F and M13R primers. All above experimental procedures were according to the methods described by Li et al. [31]. After removing the vector and primer sequences, CLUSTAL_X [36] was used to compare the DNA sequences of the two parents and the original sequences.

\section{Additional files}

\begin{abstract}
Additional file 1: Details of the $\mathbf{3 5 6}$ interspecific EST-SNP markers. SNP primer names, forward and reverse primer sequences, reference sequences used to design primers, and details of the interspecific SNPs
\end{abstract} are all listed.

Additional file 2: Primary screening process of clusters used to develop interspecific EST-SNP markers. Four types of clusters produced after identification of interspecific SNPs using HaploSNPer.

Additional file 3: Flowchart of developing inter/hemi-SNPs. i: Primers amplifying inter/hemi-SNPs; h: Primers amplifying only hemi-SNPs. One hundred and thirty-four primers amplifying inter/hemi-SNPS, and 15 primers amplifying only hemi-SNPs were developed finally.

Additional file 4: Flowchart of developing hemi-SNPs. h: Primers amplifying only hemi-SNPs. Two hundred and seven primers amplifying only hemi-SNPS were developed finally.

Additional file 5: Details of the $\mathbf{4 5 5}$ intraspecific EST-SNP markers. SNP primer names, forward and reverse primer sequences, unigenes and reference sequences used to design primers, and details of the intraspecific SNPS are all listed.

Additional file 6: Flowchart of developing intraspecific EST-SNP markers. After several steps of selection, only 8,572 of the total 21,738 unigenes were eligible for further analysis to design intraspecific EST-SNP markers, and 455 markers amplifying intraspecific EST-SNPs were developed finally.

Additional file 7: Details of the 415 EST-InDel markers developed by mining the 3 'UTRs of public $G$. hirsutum sequences. InDel primer names, forward and reverse primer sequences, reference sequences used to design primers and their Genebank numbers are all listed.

Additional file 8: Details of the 123 EST-InDel markers developed by blasting putative $3^{\prime} U T R s$ of $G$. hirsutum against the $3^{\prime} U T R s$ of Arabidopsi.s InDel primer names, forward and reverse primer sequences, and reference sequences used to design primers are all listed.

Additional file 9: Flowchart of developing HAU-InDel-prefixed markers by blasting putative 3'UTRs of G. hirsutum against the 3'UTRs of Arabidopsis. Three parts of cotton 3'UTRs were undergone blast analysis against the Arabidopsis $3^{\prime} U T R s$ respectively. Obtained unique sequences produced 62 contigs and 111 singlets, then 123 primers amplifying cotton InDels existing in $3^{\prime}$ UTRs were developed.

Additional file 10: Linkage map of 26 cotton chromosomes based on an interspecific BC $_{1}$ population. SNP and InDel markers reported in this research are italicized, underlined and bolded.

Additional file 11: SNP frequencies of cotton genes on level 3. There were 4 sub-categories in cell component category (a), 24 in molecular function category (b) and 32 in biological process category (c). Among all the sub-categories, the number of SNPs/genes varied from 1.00 to 24.00

Additional file 12: The electrophoresis gel of marker HAU-SNP572.

Competing interests

The authors declare that they have no competing interests.

Authors' contributions

XML performed most of the experiments and drafted the manuscript. WHG carried out the bioinformatics analysis of the functional annotation of sequences. HLG participated in part of the genotyping of the $B C_{1}$ population. XLZ proofed the manuscript. DDF gave many advices on the manuscript structure. ZXL designed the primers and instructed the experiments. All authors read and approved the final manuscript.

\section{Acknowledgements}

This work was financially supported by the National Basic Research Program (No. 2011CB109303) and the National Science Foundation of China (31171593). 


\section{Author details}

${ }^{1}$ National Key Laboratory of Crop Genetic Improvement \& National Centre of Plant Gene Research (Wuhan), Huazhong Agricultural University, Wuhan 430070, Hubei, China. ${ }^{2}$ College of Agronomy and Plant Protection, Qingdao Agricultural University/Shandong Key Laboratory of Dryland Farming Technology, Qingdao 266109, Shandong, China. ${ }^{3}$ Cotton Fiber Bioscience Research Unit, USDA-ARS, Southern Regional Research Center, New Orleans, LA 70124, USA.

Received: 1 June 2014 Accepted: 14 November 2014

Published: 1 December 2014

\section{References}

1. An C, Saha S, Jenkins JN, Ma DP, Scheffler BE, Kohel RJ, Yu JZ, Stelly DM: Cotton (Gossypium spp.) R2R3-MYB transcription factors SNP identification, phylogenomic characterization, chromosome localization, and linkage mapping. Theor App/ Genet 2008, 116(7):1015-1026.

2. Van Deynze A, Stoffel K, Lee M, Wilkins TA, Kozik A, Cantrell RG, Yu JZ, Kohel RJ, Stelly DM: Sampling nucleotide diversity in cotton. BMC Plant Biol 2009, 9:125.

3. Fang DD, Yu JZ: Addition of 455 microsatellite marker loci to the high-density Gossypium hirsutum TM-1 x G. barbadense 3-79 genetic map. J Cotton Sci 2012, 16:229-248.

4. Yu JZ, Kohel RJ, Fang DD, Cho J, Van Deynze A, Ulloa M, Hoffman SM Pepper AE, Stelly DM, Jenkins JN, Saha S, Kumpatla SP, Shah MR, Hugie WW Percy RG: A high-density simple sequence repeat and single nucleotide polymorphism genetic map of the tetraploid cotton genome. Gene Genome Genet 2012, 2(1):43-58.

5. Byers RL, Harker DB, Yourstone SM, Maughan PJ, Udall JA: Development and mapping of SNP assays in allotetraploid cotton. Theor App/ Genet 2012, 124(7):1201-1214

6. Lacape JM, Claverie M, Vidal RO, Carazzolle MF, Guimaraes Pereira GA, Ruiz M, Pré M, Llewellyn D, Al-Ghazi Y, Jacobs J, Dereeper A, Huguet S, Giband $M$, Lanaud C: Deep sequencing reveals differences in the transcriptional landscapes of fibers from two cultivated species of cotton. PLoS One 2012, 7(11):e48855.

7. Trick M, Long Y, Meng J, Bancroft I: Single nucleotide polymorphism (SNP) discovery in the polyploid Brassica napus using Solexa transcriptome sequencing. Plant Biotechnol J 2009, 7(4):334-346.

8. Zhu YL, Song QJ, Hyten DL, Van Tassell CP, Matukumalli LK, Grimm DR, Hyatt SM, Fickus EW, Young ND, Cregan PB: Single-nucleotide polymorphisms in soybean. Genetics 2003, 163:1123-1134.

9. Cai CM, Van K, Kim MY, Jun TH, Shin JH, Cho SY, Lee YS, Lee SH: SNP discovery, linkage analysis and microsynteny in tentative consensus sequences derived from roots CDNA in a supernodulating soybean mutant. Euphytica 2008, 164(1):189-197.

10. Chao S, Zhang W, Akhunov E, Sherman J, Ma Y, Luo MC, Dubcovsky J: Analysis of gene-derived SNP marker polymorphism in US wheat (Triticum aestivum L.) cultivars. Mol Breed 2009, 23:23-33.

11. Schmid KJ, Sörensen TR, Stracke R, Torjek O, Altmann T, Mitchell-Olds T, Weisshaar B: Large-scale identification and analysis of genome-wide single-nucleotide polymorphisms for mapping in Arabidopsis thaliana. Genome Res 2003, 13(6A):1250-1257.

12. Kota R, Rudd S, Facius A, Kolesov G, Thiel T, Zhang H, Stein N, Mayer K, Graner A: Snipping polymorphisms from large EST collections in barley (Hordeum vulgare L.). Mol Genet Genomics 2003, 270(1):24-33.

13. Batley J, Barker G, O'Sullivan H, Edwards KJ, Edwards D: Mining for single nucleotide polymorphisms and insertions/deletions in maize expressed sequence tag data. Plant Physiol 2003, 132(1):84-91.

14. Grivet L, Glaszmann JC, Vincentz M, da Silva F, Arruda P: ESTs as a source for sequence polymorphism discovery in sugarcane: example of the Adh genes. Theor App/ Genet 2003, 106(2):190-197.

15. Labate JA, Baldo AM: Tomato SNP discovery by EST mining and resequencing. Mol Breed 2005, 16(4):343-349.

16. Ferguson ME, Hearne SJ, Close TJ, Wanamaker S, Moskal WA, Town CD, de Young J, Marri PR, Rabbi IY, de Villiers EP: Identification, validation and high-throughput genotyping of transcribed gene SNPs in cassava. Theor Appl Genet 2012, 124(4):685-695.

17. Chaisan T, Van K, Kim MY, Kim KD, Choi BS, Lee SH: In silico single nucleotide polymorphism discovery and application to marker-assisted selection in soybean. Mol Breed 2012, 29(1):221-233.
18. Picoult-Newberg L, Ideker TE, Pohl MG, Taylor SL, Donaldson MA, Nickerson DA, Boyce-Jacino M: Mining SNPs from EST databases. Genome Res 1999, 9(2):167-174.

19. Yu Y, Yuan D, Liang S, Li X, Wang X, Lin Z, Zhang X: Genome structure of cotton revealed by a genome-wide SSR genetic map constructed from a BC1 population between gossypium hirsutum and $\mathrm{G}$. barbadense. BMC Genomics 2011, 12:15.

20. Mouse Genome Sequencing Consortium, Waterston RH, Lindblad-Toh K, Birney E, Rogers J, Abril JF, Agarwal P, Agarwala R, Ainscough R, Alexandersson M, An P, Antonarakis SE, Attwood J, Baertsch R, Bailey J, Barlow K, Beck S, Berry E, Birren B, Bloom T, Bork P, Botcherby M, Bray N, Brent MR, Brown DG, Brown SD, Bult C, Burton J, Butler J, Campbell RD, et al: Initial sequencing and comparative analysis of the mouse genome. Nature 2002, 420(6915):520-562.

21. Andreassen R, Lunner S, Høyheim B: Targeted SNP discovery in Atlantic salmon (Salmo salar) genes using a 3'UTR-primed SNP detection approach. BMC Genomics 2010, 11:706.

22. Koepke T, Schaeffer S, Krishnan V, Jiwan D, Harper A, Whiting M, Oraguzie N, Dhingra A: Rapid gene-based SNP and haplotype marker development in non-model eukaryotes using 3'UTR sequencing. BMC Genomics 2012, 13:18.

23. Guo W, Wang W, Zhou B, Zhang T: Cross-species transferability of G. arboreum-derived EST-SSRs in the diploid species of Gossypium. Theor Appl Genet 2006, 112(8):1573-1581.

24. Cao ZF, Ma CX, Wang L, Cai B: Analysis of population stratification using random SNPs in genome-wide association studies. Hereditas 2010, 32(9):921-928

25. Gupta P, Idris A, Mantri S, Asif MH, Yadav HK, Roy JK, Tuli R, Mohanty CS, Sawant SV: Discovery and use of single nucleotide polymorphic (SNP) markers in Jatropha curcas L. Mol Breed 2012, 30(3):1325-1335.

26. Geng R, Chang H, Li Y, Ji D, Chang C, Wang L, Chang G: Variation of complete coding sequence of $\mathrm{GH}$ gene in Chinese Bovinae species. Acta Vet Zootech Sin 2008, 39(12):1779-1784.

27. Tsaftaris AS, Polidoros AN: DNA methylation and plant breeding Plant Breed Rev 1999, 18:87-176.

28. Hazelhurst S, Hide W, Lipták Z, Nogueira R, Starfield R: An overview of the wcd EST clustering tool. Bioinformatics 2008, 24(13):1542-1546.

29. Wang X, Ren G, Li X, Tu J, Lin Z, Zhang X: Development and evaluation of intron and insertion-deletion markers for Gossypium barbadense. Plant Mol Biol Rep 2011, 30(3):605-613.

30. Lin Z, He D, Zhang X, Nie Y, Guo X, Feng C, Stewart JM: Linkage map construction and mapping QTL for cotton fibre quality using SRAP, SSR and RAPD. Plant Breed 2005, 124:180-187.

31. Li $X$, Yuan D, Wang $H$, Chen $X$, Wang $B$, Lin Z, Zhang $X$ : Increasing cotton genome coverage with polymorphic SSRs as revealed by SSCP. Genome 2012, 55(6):459-470.

32. Liu C, Lin Z, Zhang X: Unbiased genomic distribution of genes related to cell morphogenesis in cotton by chromosome mapping. Plant Cell Tiss Org 2012, 108(3):529-534.

33. Conesa A, Gotz S, Garcia-Gomez JM, Terol J, Talón M, Robles M: Blast2GO: a universal tool for annotation, visualization and analysis in functional genomics research. Bioinformatics 2005, 21(18):3674-3676.

34. Gotz S, Garcia-Gomez JM, Terol J, Williams TD, Nagaraj SH, Nueda MJ, Robles M, Talón M, Dopazo J, Conesa A: High-throughput functional annotation and data mining with the Blast2GO suite. Nucleic Acids Res 2008, 36(10):3420-3435.

35. Li X, Yuan D, Zhang J, Lin Z, Zhang X: Genetic mapping and characteristics of genes specifically or preferentially expressed during fiber development in cotton. PLoS One 2013, 8(1):e54444.

36. Thompson JD, Gibson TJ, Plewniak F, Jeanmougin F, Higgins DG: The CLUSTAL_X windows interface: flexible strategies for multiple sequence alignment aided by quality analysis tools. Nucleic Acids Res 1997, 25(24):4876-4882.

doi:10.1186/1471-2164-15-1046

Cite this article as: Li et al.: Development of EST-based SNP and InDel markers and their utilization in tetraploid cotton genetic mapping. BMC Genomics 2014 15:1046. 